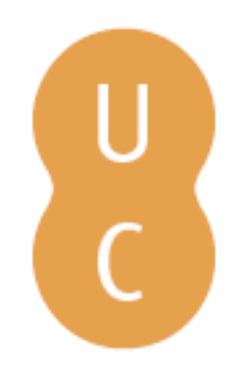

\title{
pommalina
}

\section{O impacto da produção científica e padrões de citação entre pesquisadores do} Centro de Pesquisa
Autor(es):
Santana, Celeste Maria de Oliveira; Sampaio, Ana Maria Fiscina Vaz; Morte, Adelvani de Araújo Boa; Martínez-Silveira, Martha Silvia; Rocha, Anderson Silva da

Publicado por: Imprensa da Universidade de Coimbra

URL persistente:

URI:http://hdl.handle.net/10316.2/31971

DOI:

DOl:http://dx.doi.org/10.14195/978-989-26-0869-3_44

Accessed : $\quad$ 26-Apr-2023 07:17:44

A navegação consulta e descarregamento dos títulos inseridos nas Bibliotecas Digitais UC Digitalis, UC Pombalina e UC Impactum, pressupõem a aceitação plena e sem reservas dos Termos e Condições de Uso destas Bibliotecas Digitais, disponíveis em https://digitalis.uc.pt/pt-pt/termos.

Conforme exposto nos referidos Termos e Condições de Uso, o descarregamento de títulos de acesso restrito requer uma licença válida de autorização devendo o utilizador aceder ao(s) documento(s) a partir de um endereço de IP da instituição detentora da supramencionada licença.

Ao utilizador é apenas permitido o descarregamento para uso pessoal, pelo que o emprego do(s) título(s) descarregado(s) para outro fim, designadamente comercial, carece de autorização do respetivo autor ou editor da obra.

Na medida em que todas as obras da UC Digitalis se encontram protegidas pelo Código do Direito de Autor e Direitos Conexos e demais legislação aplicável, toda a cópia, parcial ou total, deste documento, nos casos em que é legalmente admitida, deverá conter ou fazer-se acompanhar por este aviso.

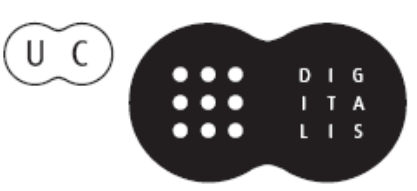


Maria Manuel Borges

Elias Sanz Casado

Coordenação

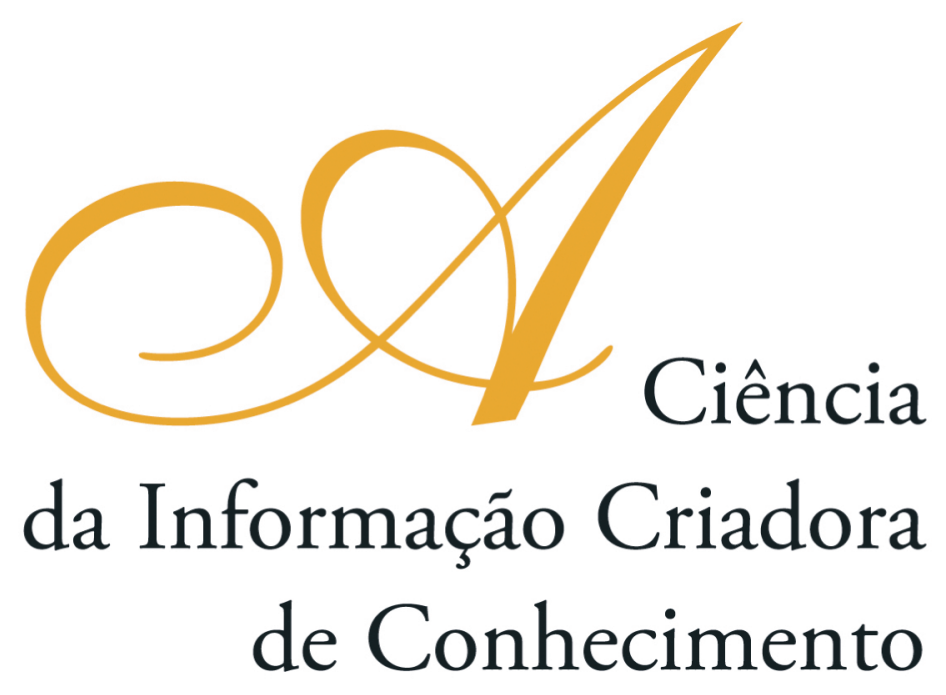

Vol. I I

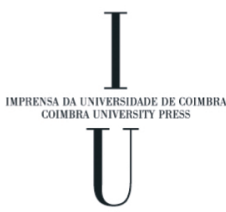

- COImbra 2009 


\section{O impacto da produção científica e padróes de citação entre pesquisadores do Centro de Pesquisa}

\section{Celeste Maria de Oliveira Santana}

Universidade Federal da Bahia (Brasil)

Ana Maria Fiscina Vaz Sampaio

Casa de Oswaldo Cruz (Brasil)

Adelvani de Araújo Boa Morte

Casa de Oswaldo Cruz (Brasil)

Martha Silvia Martínez-Silveira

Casa de Oswaldo Cruz (Brasil)

Anderson Silva da Rocha

Casa de Oswaldo Cruz (Brasil)

\section{Resumo}

A publicação de artigos da comunidade científica do CPqGM tem como principal finalidade a comunicação dos resultados das pesquisas e divulgação da sua produção, buscando atingir o reconhecimento dos seus pares. A publicação é um dos elementos mais importantes na avaliação dos programas de pós-graduação e pesquisa no Brasil, sendo o estudo do impacto dessa publicaçáo importante como meio de avaliação da qualidade da produçáo de uma instituição. Este estudo teve lugar num centro de excelência no estado da Bahia na área de doenças infectoparasitárias onde se concentram pesquisadores do mais alto conceito. A pesquisa originada em dados obtidos em etapa anterior (2005), mapeia e quantifica a produçáo científica a partir da análise bibliométrica dos artigos publicados em periódicos nacionais e internacionais entre os anos 2000 e 2007. OBJETIVO: Verificar o impacto da publicaçâo produzida no CPqGM a partir dos indicadores (Fator de Impacto, Fator H e Qualis) e da análise de citaçôes dos artigos dos pesquisadores entre si. METODOLOGIA: Todos os pesquisadores vinculados ao Centro foram incluídos. As fontes foram o Curriculum Lattes, Cadernos CAPES, Medline, Lilacs e Scielo. Foi elaborado um banco de dados dos artigos publicados, feita análise bibliométrica que incluiu: número de artigos, FI e Qualis dos periódicos, número de citações por artigo, fator $\mathrm{H}$ dos pesquisadores e dos artigos, número de periódicos indexados nas bases de dados, e análise das citaçôes entre os próprios pesquisadores do Centro. RESULTADOS PRELIMINARES: São 30 pesquisadores selecionados que publicaram isoladamente ou em conjunto 541 artigos em 168 revistas internacionais e 50 revistas nacionais entre 2000 e 2007. Ao final desta pesquisa serão gerados dados sobre as equipes mais produtivas e mais citadas, temas sobre os quais mais se publica, nível de endogenicidade da produtividade do Centro. Estes dados poderão servir de informação para as agencias de fomento à pesquisa e para a própria instituição. 


\begin{abstract}
The publication of papers from the scientific community of the CPqGM has, as its main objective, the communication of results and divulgation of its production, aiming at recognition by pairs. Publication is one of the most important elements on the evaluation of the post-graduate and research programs in Brazil. Therefore, an study about the impact of such publication is important as a way of assessing the quality of the production at an institution. This study took place at an excellence center in the state of Bahia, in the area of infectious-parasitary diseases, where there is a concentration of highly important researchers. The research based on data obtained from a previous phase (2005), maps and quantifies the scientific production from the bibliometric analysis of the published papers in national and international journals between 2000 to 2007. OBJECTIVE: To verify the impact of publication produced at the CPqGM from certain indicators (Impact Factor, H Factor and Qualis) and the analysis of paper and researcher citations. METHODOLOGY: All researchers formally working at the Center were included. The sources were Curriculum Lattes, Cadernos CAPES, Medline, Lilacs and Scielo. A data base of the published articles was elaborated and a bibliometric analysis was made, which included: number of papers, IF and Qualis of the journals, number of citations by paper, $\mathrm{H}$ Factor of researchers and papers, number of indexed papers on known data bases and analysis of citation among researchers from the Center. PRELIMINARY RESULTS: There are 30 selected researchers who published, individually or together with others authors, 541 articles in 168 international journals and 50 national journals between 2000 and 2007. At the end of this research, data will be generated about the most productive and most cited teams, which are the most published themes and the level of endogenicity of the Center's productivity. This data could be used as a source of information for the agencies of support to research and the institution itself.
\end{abstract}

\title{
Introdução
}

A pesquisa foi originada de dados obtidos em etapa anterior (Parte I) apresentado no ICML9 (2005) intitulado A publicação de "papers" nas revistas científicas que causam impacto em uma comunidade científica na área de doenças infecciosas e parasitárias, no período de 2000 a 2004 . Naquele trabalho foram mapeados os artigos dos pesquisadores do Centro de Pesquisa Gonçalo Moniz (CPqGM) da Fundaçáo Oswaldo Cruz (FIOCRUZ) no estado da Bahia, durante o período de 2000 a 2004.

O presente trabalho (Parte II) visa atualizar esse mapeamento até o ano de 2007 com a finalidade de quantificar a produção científica dos Pesquisadores, e a partir da análise bibliométrica dos artigos publicados demonstrar a visibilidade da produçáo científica originada nos laboratórios de pesquisa dessa instituição.

O CPqGM é uma unidade da Fiocruz localizada no estado da Bahia dedicada principalmente ao estudo das doenças infecciosas e parasitárias de maior impacto na população brasileira. O CPqGM, centro de referência em saúde, além de suas açóes na área biomédica, e de desenvolver recursos humanos para o SUS, mantém cursos de pós-graduação do mais alto nível de excelência. Os cursos de Patologia e Biotecnologia em Saúde e Medicina Investigativa reúnem pesquisadores e alunos cuja produção científica destaca-se no cenário nacional e internacional.

As atividades se organizam em dez laboratórios e outras unidades: LACEI Laboratório de Chagas Experimental; LAPEX - Laboratório da Patologia Experimental; LASP - Laboratório Avançado de Saúde Pública; LBP - Laboratório de Biomorfologia 
Parasitária; LEMB - Laboratório de Epidemiologia Molecular e Bioestatística; LETI - Laboratório de Engenharia Tecidual e Imunofarmacologia; LIMI - Laboratório Integrado de Microbiologia e Imunoregulação; LIP - Laboratório de Imunoparasitologia; LPBI - Laboratório de Patologia e Biointervenção; LPBM - Laboratório de Patologia e Biologia Molecular e o Serviço de Histopatologia.

\section{Objetivo}

Verificar o impacto da produção científica produzida pela comunidade do CPqGM e publicada em artigos internacionais e nacionais a partir dos indicadores bibliométricos como o Fator de Impacto, Fator H e Qualis (CAPES) e da análise de citaçóes dos artigos e dos pesquisadores entre si.

\section{Metodologia}

O estudo de caso foi realizado especificamente, com os Pesquisadores do CPqGM que são vinculados à FIOCRUZ/BAHIA tomando-se como unidade a análise dos artigos científicos publicados por esta população. A cada pesquisador foi dado um código numérico para que se pudessem efetuar as análises de forma anônima.

Inicialmente, foi elaborado um banco de dados contendo as informaçóes dos artigos publicados divididos em nacionais e internacionais. As fontes de coleta de dados foram o Curriculum Lattes, os Cadernos CAPES, as listas da Coordenção de Ensino do CPqGM, as bases de dados bibliográficas Medline, Scopus, LILACS e as páginas do site do CPqGM para obter as informaçóes referentes aos artigos publicados. Para as análises de citaçóes e o impacto dos periódicos foram utilizados o Journal of Citation Report (JCR), o Qualis e os dados estatísticos do Scientific Electronic Library On Line (SciELO) assim como, as referências bibliográficas de cada artigo.

\section{Resultados}

De acordo com o critério estabelecido foram selecionados 30 pesquisadores que publicaram isoladamente, ou em conjunto 541 artigos em revistas internacionais e nacionais durante o período de 2000 a 2007. Foram publicados 329 artigos em periódicos internacionais e 212 artigos em periódicos nacionais (Fig. 1). 


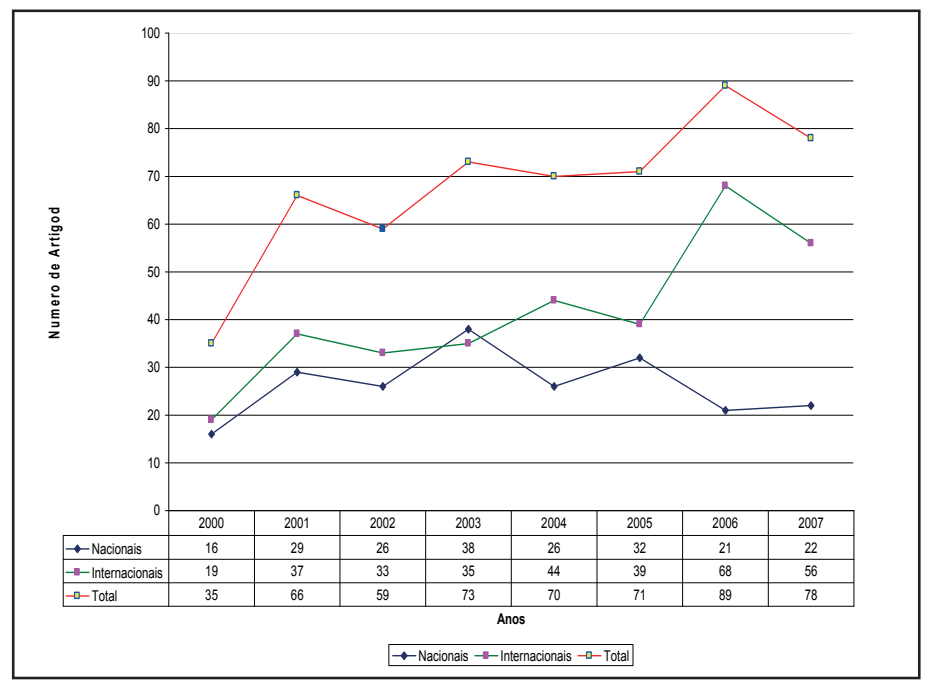

Fig. 1 - Artigos publicados pelos pesquisadores do CPqGM entre os anos de 2000 e 2007

Analisados por Laboratórios, percebeu-se que o Laboratório que mais publicou artigos nacionais foi o LAPEX (50), enquanto que o LPBI publicou mais Artigos internacionais (61). Na sua totalidade o LIP foi o que mais publicou (89) (Fig. 2). Levando-se em consideração o número de pesquisadores por Laboratório, observouse que os pesquisadores mais profusos estavam no LAPEX, com uma média de 39.5 artigos (Fig. 3).

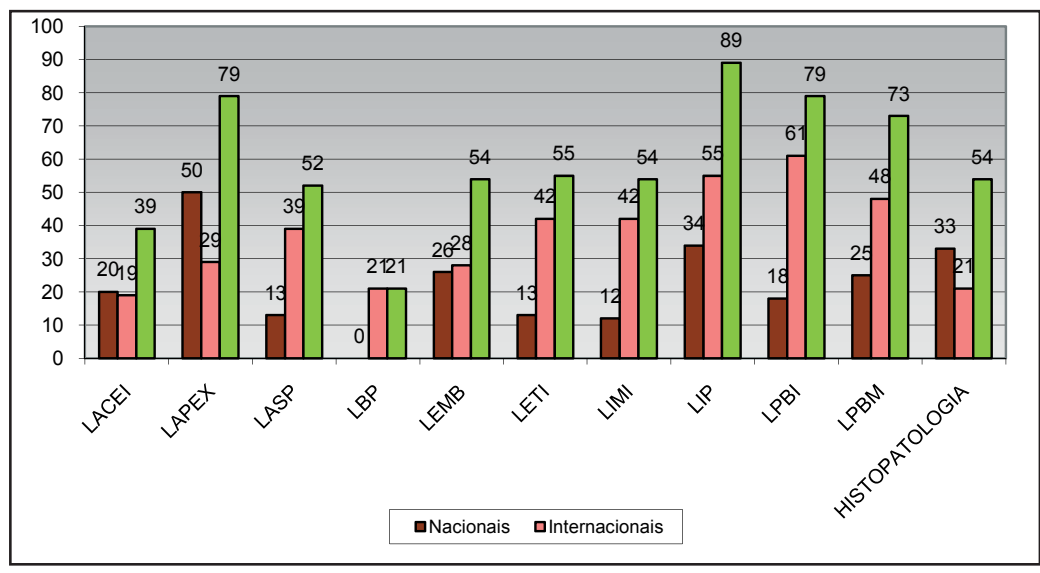

Fig. 2 - Número de Artigos por Laboratórios do CPqGM entre os anos de 2000 e 2007 


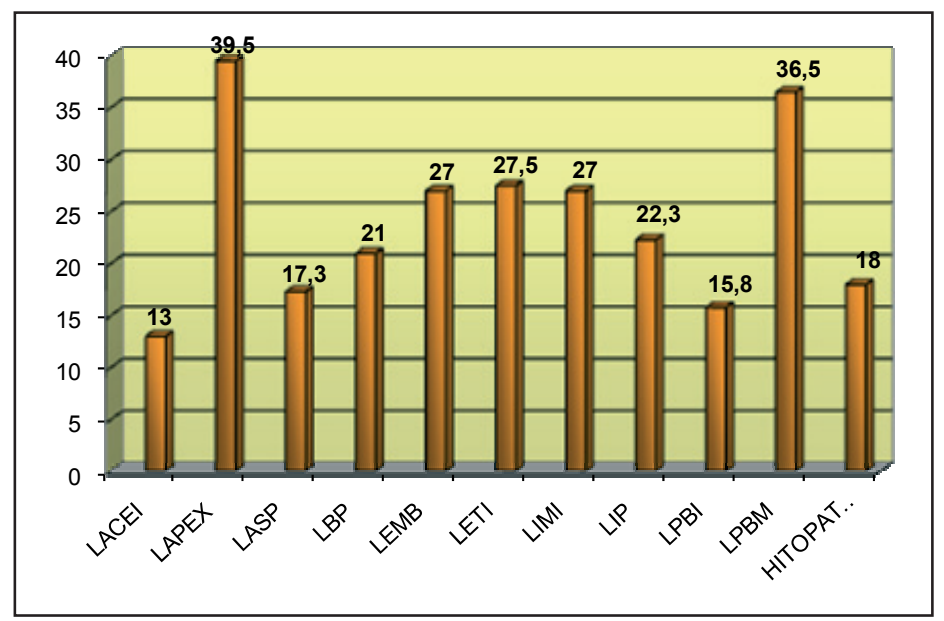

Fig. 3 - Média de artigos publicados pelos Pesquisadores do CPqGM entre os anos 2000 e 2007

O fator $\mathrm{H}$ dos pesquisadores, foi levantado na base de dados Scopus, onde se verificou que o maior número atingido foi $h$-index 16 , de pesquisadores locados nos laboratórios LEMB, LIMI e LIPI. Pos sua vez, os pesquisadores que mais publicaram foram, a nível nacional do laboratório LAPEX (50), internacional LPBM (44) e em sua totalidade o pesquisador locado no LAPEX (71) (Tabela 1). A análise das citaçóes e de auto-citaçóes considerou apenas aquelas que constavam nos trabalhos dos pesquisadores que estavam sendo analisados. Para a realização deste trabalho, foi extraído de cada artigo o número de referências onde ao menos um dos pesquisadores do Centro tivesse sido citado. Estes resultados preliminares estão também representados na Tabela 1.

Tabela 1 - Avaliação da Produção Científica dos Pesquisadores do CPqGM

\begin{tabular}{|l|l|l|l|l|l|l|l|}
\hline $\begin{array}{l}\text { Cód. } \\
\text { Pesq. }\end{array}$ & Laboratórios & Fator H & Citaçóes & $\begin{array}{l}\text { Auto- } \\
\text { Citaçóes }\end{array}$ & Art. Inter. & Art. Nac. & $\begin{array}{l}\text { Total de } \\
\text { Artigos }\end{array}$ \\
\hline 1 & LIP & 16 & 41 & 112 & 43 & 13 & 56 \\
\hline 2 & LPBI & 0 & 0 & 0 & 0 & 0 & 0 \\
\hline 3 & HISTOPATOL. & 3 & 2 & 9 & 9 & 13 & 32 \\
\hline 4 & LASP & 12 & 13 & 44 & 34 & 11 & 45 \\
\hline 5 & LIP & 11 & 16 & 23 & 25 & 1 & 26 \\
\hline 6 & LEMB & 16 & 1 & 43 & 27 & 22 & 49 \\
\hline 7 & HISTOPATOL. & 2 & 1 & 2 & 8 & 9 & 17 \\
\hline 8 & LACEI & 3 & 1 & 20 & 8 & 0 & 8 \\
\hline 9 & LASP & 4 & 0 & 0 & 3 & 1 & 4 \\
\hline 10 & LPBI & 4 & 3 & 3 & 6 & 1 & 7 \\
\hline 11 & LIP & 9 & 10 & 13 & 17 & 23 & 40 \\
\hline 12 & LIMI & 6 & 0 & 3 & 11 & 0 & 11 \\
\hline 13 & LIP & 6 & 4 & 4 & 8 & 1 & 9 \\
\hline 14 & LACEI & 4 & 17 & 24 & 14 & 1 & 15 \\
\hline 15 & LPBI & 10 & 26 & 48 & 29 & 5 & 34 \\
\hline
\end{tabular}




\begin{tabular}{|l|l|l|l|l|l|l|l|}
\hline 16 & LAPEX & 1 & 4 & 6 & 8 & 0 & 8 \\
\hline 17 & LASP & 5 & 4 & 20 & 16 & 5 & 21 \\
\hline 18 & LPBI & 4 & 22 & 27 & 27 & 11 & 38 \\
\hline 19 & LEMB & 0 & 4 & 0 & 1 & 4 & 5 \\
\hline 20 & LIMI & 16 & 62 & 72 & 38 & 12 & 50 \\
\hline 21 & LBP & 11 & 1 & 59 & 21 & 0 & 21 \\
\hline 22 & LPBM & 9 & 7 & 8 & 10 & 11 & 21 \\
\hline 23 & LETI & 13 & 21 & 30 & 35 & 12 & 47 \\
\hline 24 & LPBM & 12 & 7 & 51 & 44 & 21 & 65 \\
\hline 25 & LPBI & 2 & 4 & 5 & 6 & 0 & 6 \\
\hline 26 & LETI & 9 & 19 & 31 & 26 & 12 & 38 \\
\hline 27 & HISTOPATOL. & 4 & 0 & 0 & 4 & 2 & 6 \\
\hline 28 & LACEI & 9 & 18 & 21 & 3 & 19 & 22 \\
\hline 29 & LPBI & 6 & 10 & 28 & 16 & 5 & 21 \\
\hline 30 & LAPEX & 11 & 24 & 61 & 21 & 50 & 71 \\
\hline
\end{tabular}

Os artigos foram publicados em 168 revistas internacionais e 50 nacionais, sendo que na revista Memórias do Instituto Oswaldo Cruz foram publicados 48 artigos e a revista internacional com mais artigos dos pesquisadores foi a American Journal of Tropical Medicine and Hygiene com 15 artigos (Tabelas 2 e 3). Os periódicos internacionais onde se encontra a maior quantidade de artigos publicados pelos pesquisadores do Centro tem um fator de impacto segundo o JCR que varia entre os valores 1 e 2 (Fig. 4). Enquanto que os nacionais tem um fator de impacto analisado pelo SciELO entre 0,05 e 0,2 (Fig. 5). A avaliação Qualis dos periódicos nacionais e internacionais se encontra representada no Fig. 6.

Tabela 2 - Periódicos Nacionais onde os Pesquisadores do CPqGM mais publicaram entre os anos de 2000 e 2007

\begin{tabular}{|l|l|l|}
\hline Periódicos Nacionais & $\begin{array}{l}\text { Num } \\
\text { Artigos }\end{array}$ & $\begin{array}{l}\text { FI } \\
\text { Scielo }\end{array}$ \\
\hline Memórias do Instituto Oswaldo Cruz & 48 & 0.3756 \\
\hline Revista da Sociedade Brasileira de Medicina Tropical & 39 & 0.4366 \\
\hline Brazilian Journal of Medical and Biological Research & 10 & 0.1862 \\
\hline Cadernos de Saúde Pública (FIOCRUZ) & 10 & 0.6540 \\
\hline Revista Brasileira de Medicina & 8 & - \\
\hline Gazeta Médica da Bahia & 7 & - \\
\hline Jornal Brasileiro de Patologia e Medicina Laboratorial & 7 & 0.0593 \\
\hline Anais Brasileiros de Dermatologia & 6 & 0.1379 \\
\hline Revista de Ciências Médicas e Biológicas & 6 & - \\
\hline Brazilian Journal of Infectious Diseases & 5 & 0.1788 \\
\hline Ciência e Saúde (Salvador) & 5 & - \\
\hline Revista de Patologia Tropical & 5 & - \\
\hline Revista do Hospital Universitário (UFMA) & 5 & - \\
\hline Revista Brasileira de Hematologia e Hemoterapia & 4 & 0.0935 \\
\hline
\end{tabular}


Tabela 3 - Periódicos Internacionais onde os Pesquisadores do CPqGM mais publicaram entre os anos de 2000 e 2007

\begin{tabular}{|l|l|l|}
\hline Periódicos Internacionais & $\begin{array}{l}\text { Quantidade de } \\
\text { Artigos }\end{array}$ & $\begin{array}{l}\text { FI } \\
\text { JCR }\end{array}$ \\
\hline American Journal of Tropical Medicine and Hygiene & 15 & 2.183 \\
\hline Infection and Immunity & 15 & 3.996 \\
\hline Acta Tropica & 12 & 2.000 \\
\hline Microbes and Infection & 10 & 2.523 \\
\hline AIDS Research and Human Retroviruses & 9 & 2.022 \\
\hline Journal of Acquired Immune Deficiency Syndromes & 8 & 3.946 \\
\hline Parasitology Research & 8 & 1.512 \\
\hline Transactions of the Royal Society of Tropical Medicine and Hygiene & 7 & 1.924 \\
\hline International Journal of Impotence Research & 6 & 2.059 \\
\hline Journal of Infectious Diseases & 6 & 6.035 \\
\hline Experimental Parasitology & 5 & 1.597 \\
\hline Gynecological Endocrinology & 5 & 1.169 \\
\hline Scandinavian Journal of Immunology & 5 & 1.928 \\
\hline Veterinary Parasitology & 5 & 2.016 \\
\hline Hybridoma and Hybridomics & 4 & 0.294 \\
\hline Journal of Clinical Microbiology & 4 & 3.708 \\
\hline Pathology Research and Practice & 4 & 1.080 \\
\hline Urology & 4 & 2.134 \\
\hline Vaccine & 4 & 3.377 \\
\hline
\end{tabular}

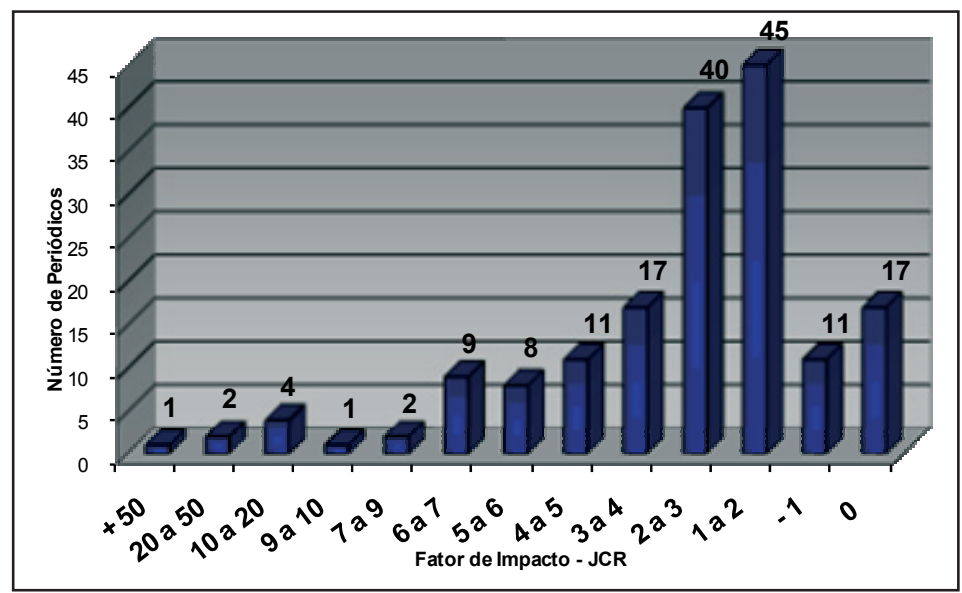

Fig. 4 - Fator de Impacto (JCR) dos Periódicos Internacionais 


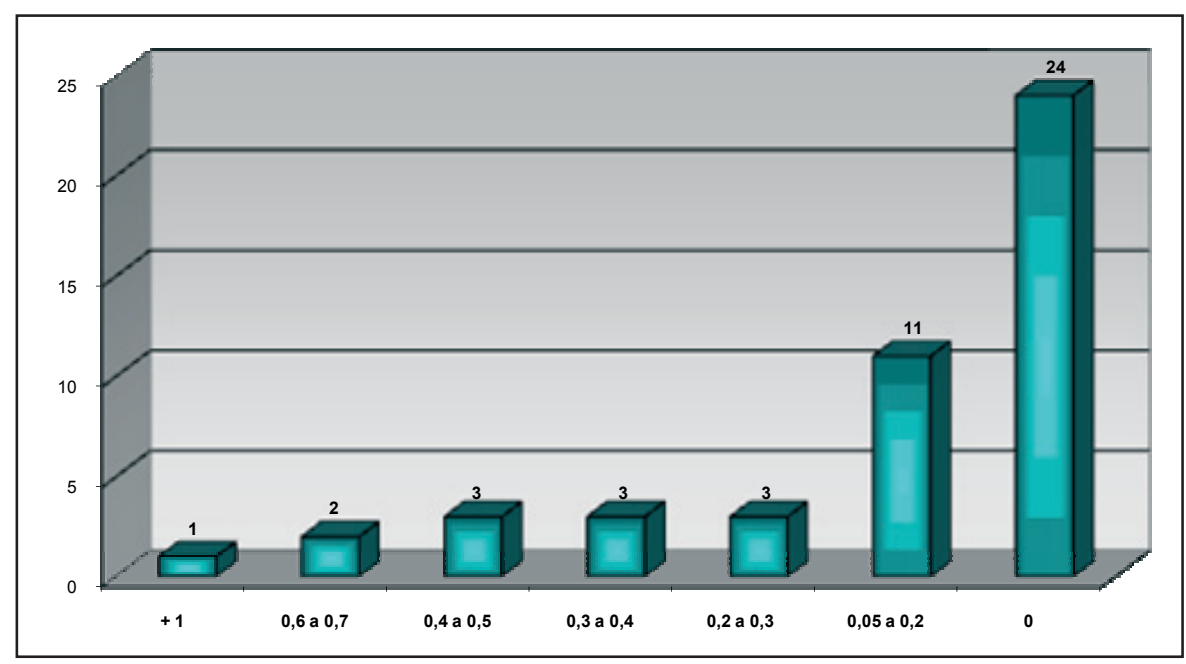

Fig. 5 - Fator de Impacto (SciELO) dos Periódicos Nacionais

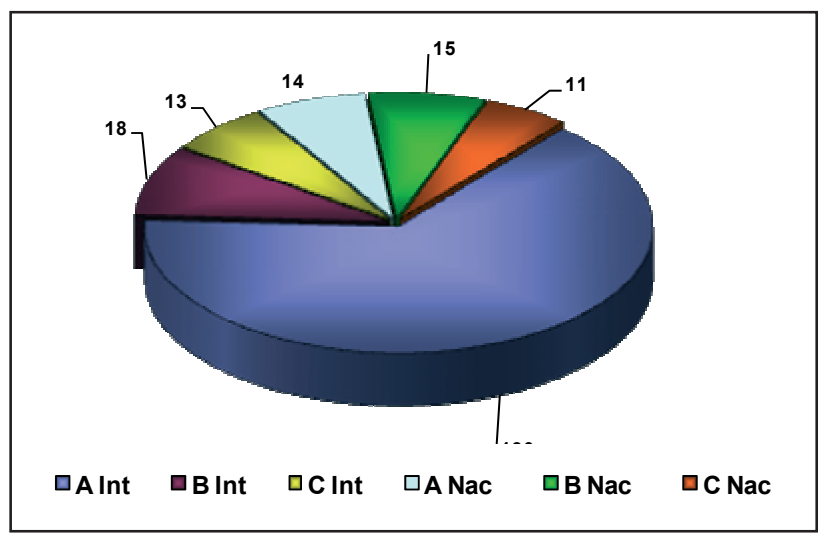

Fig. 6 - Classificação QUALIS dos Periódicos Nacionais e Internacionais onde publicaram os Pesquisadores do CPqGM

Os artigos nacionais e internacionais que obtiveram maior número de citaçôes nas bases de dados Web of Science e Scopus estáo relacionados nas Tabelas 4 e 5. 
Tabela 4 - Artigos Internacionais e Nacionais mais citados na Base de Dados Web OF SCIENCE

\begin{tabular}{|l|l|l|l|}
\hline Cód. Autores & Periódico & Ano & $\begin{array}{l}\text { No citaçóes } \\
\text { Web of Science }\end{array}$ \\
\hline 23 & Nature & 2000 & 179 \\
\hline 6 & International Journal of Impotence Research & 2005 & 129 \\
\hline 6 & New England Journal of Medicine & 2007 & 110 \\
\hline 6 & Urology & 2003 & 103 \\
\hline 4 & AIDS Research and Human Retroviruses & 2001 & 88 \\
\hline 4 & Journal of Acquired Immune Deficiency Syndromes & 2000 & 50 \\
\hline 24 & Current opinion in infectious diseases & 2005 & 46 \\
\hline 6 & Urology & 2004 & 46 \\
\hline 8 & Journal of Immunology & 2004 & 41 \\
\hline 24 & Human Immunology & 2000 & 40 \\
\hline 6 & Urology & 2001 & 40 \\
\hline $15,23,26$ & Anais da Academia Brasileira de Ciências & 2001 & 31 \\
\hline 4 & Memórias do Instituto Oswaldo Cruz & 2002 & 20 \\
\hline $1,13,20,22,24$ & Memórias do Instituto Oswaldo Cruz & 2003 & 20 \\
\hline 30 & Memórias do Instituto Oswaldo Cruz & 2002 & 16 \\
\hline $18,20,27$ & Memórias do Instituto Oswaldo Cruz & 2001 & 15 \\
\hline 1,20 & Memórias do Instituto Oswaldo Cruz & 2003 & 14 \\
\hline 1,20 & Memórias do Instituto Oswaldo Cruz & 2000 & 12 \\
\hline 4 & Memórias do Instituto Oswaldo Cruz & 2003 & 11 \\
\hline $1,20,22,24$ & Brazilian Journal of Medical \& Biological Research & 2001 & 9 \\
\hline 15,29 & Memórias do Instituto Oswaldo Cruz & 2004 & 9 \\
\hline 11 & Brazilian Journal of Medical \& Biological Research & 2004 & 8 \\
\hline $15,23,26$ & Anais da Academia Brasileira de Ciências & 2001 & 31 \\
\hline 4 & Memórias do Instituto Oswaldo Cruz & 2002 & 20 \\
\hline & & & \\
\hline & & & \\
\hline
\end{tabular}

Tabela 5 - Artigos internacionais e nacionais mais citados na Base de Dados SCOPUS

\begin{tabular}{|l|l|l|l|}
\hline Cód. Autores & Periódico & Ano & $\begin{array}{l}\text { No citaçóes } \\
\text { Scopus }\end{array}$ \\
\hline 23 & Nature & 2000 & 192 \\
\hline 6 & International Journal of Impotence Research & 2005 & 147 \\
\hline 6 & New England Journal of Medicine & 2007 & 140 \\
\hline 6 & Urology & 2003 & 124 \\
\hline 4 & AIDS Research and Human Retroviruses & 2001 & 64 \\
\hline 24 & Journal of Clinical Microbiology & 2001 & 53 \\
\hline 6 & Urology & 2001 & 53 \\
\hline 24 & Current opinion in infectious diseases & 2005 & 52 \\
\hline 7 & Journal of pediatric and perinatal nutrition & 2003 & 50 \\
\hline 24 & Infection and Immunity & 2005 & 48 \\
\hline 4 & Journal of Acquired Immune Deficiency Syndromes & 2000 & 44 \\
\hline $15,23,26$ & Anais da Academia Brasileira de Ciências & 2001 & 35 \\
\hline 19 & Cadernos de Saúde Pública & 2003 & 29 \\
\hline
\end{tabular}




\begin{tabular}{|l|l|l|l|}
\hline 1,20 & Memórias do Instituto Oswaldo Cruz & 2000 & 24 \\
\hline 4 & Memórias do Instituto Oswaldo Cruz & 2002 & 20 \\
\hline 1,20 & Memórias do Instituto Oswaldo Cruz & 2003 & 19 \\
\hline 6 & Sáo Paulo Medical Journal & 2002 & 19 \\
\hline $1,13,20,22,24$ & Memórias do Instituto Oswaldo Cruz & 2003 & 17 \\
\hline $18,20,27$ & Memórias do Instituto Oswaldo Cruz & 2001 & 15 \\
\hline $22,24,28$ & Revista da Sociedade Brás. de Medicina Tropical & 2003 & 12 \\
\hline 4 & Memórias do Instituto Oswaldo Cruz & 2003 & 11 \\
\hline 19 & Revista de Saúde Pública & 2003 & 11 \\
\hline
\end{tabular}

Em relação aos temas abordados nos artigos encontram-se representados nas Figs. 7 e 8.

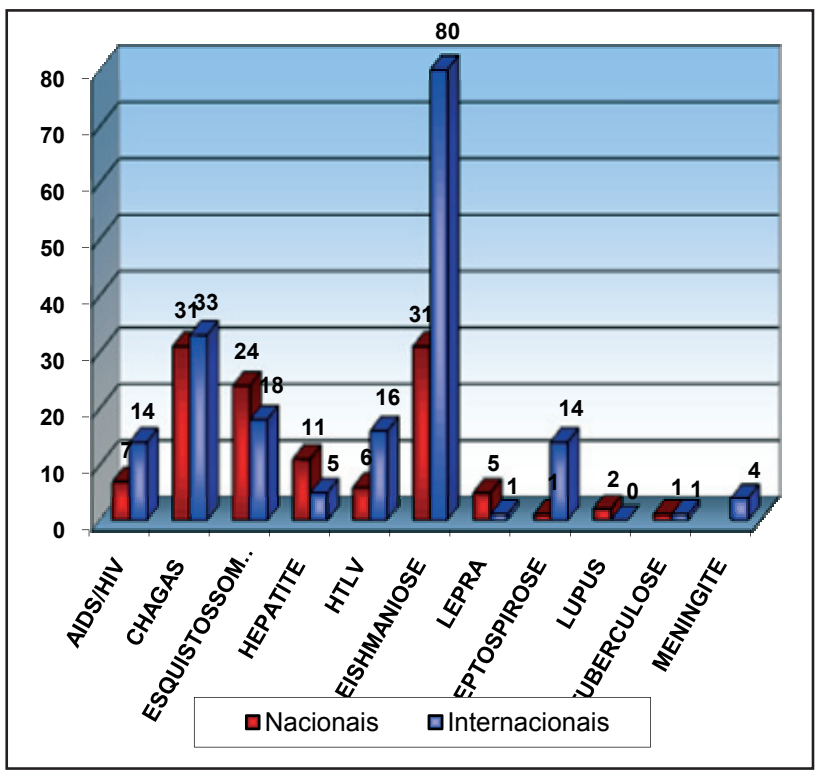

Fig. 7 - Principais Temas pesquisados no CPqGM e o número de Artigos Nacionais e Internacionais publicados entre os anos de 2000 e 2007 


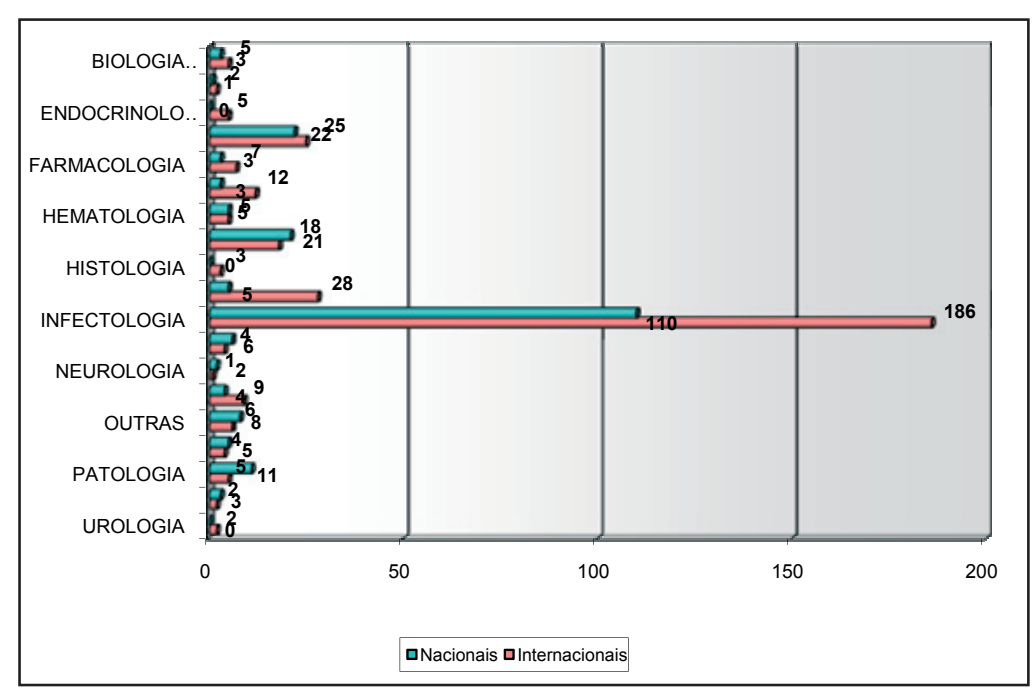

Fig. 8 - Temas dos Artigos publicados no CPqGM divididos em grandes Áreas entre os anos de 2000 e 2007

Os artigos mais citados nas bases de dados SCOPUS e WEB OF SCIENCE foram relacionados nas Tabelas 6 e 7 .

Tabela 6 - Artigos Internacionais e Nacionais mais citados na Base de Dados WEB OF SCIENCE

\begin{tabular}{|l|l|l|l|}
\hline Cód. Autores & Periódico & Ano & $\begin{array}{l}\text { No citaçôes } \\
\text { Web of Science }\end{array}$ \\
\hline 23 & Nature & 2000 & 179 \\
\hline 6 & International Journal of Impotence Research & 2005 & 129 \\
\hline 6 & New England Journal of Medicine & 2007 & 110 \\
\hline 6 & Urology & 2003 & 103 \\
\hline 4 & AIDS Research and Human Retroviruses & 2001 & 88 \\
\hline 4 & Journal of Acquired Immune Deficiency Syndromes & 2000 & 50 \\
\hline 24 & Current opinion in infectious diseases & 2005 & 46 \\
\hline 6 & Urology & 2004 & 46 \\
\hline 8 & Journal of Immunology & 2004 & 41 \\
\hline 24 & Human Immunology & 2000 & 40 \\
\hline 6 & Urology & 2001 & 40 \\
\hline $15,23,26$ & Anais da Academia Brasileira de Ciências & 2001 & 31 \\
\hline 4 & Memórias do Instituto Oswaldo Cruz & 2002 & 20 \\
\hline $1,13,20,22,24$ & Memórias do Instituto Oswaldo Cruz & 2003 & 20 \\
\hline 30 & Memórias do Instituto Oswaldo Cruz & 2002 & 16 \\
\hline $18,20,27$ & Memórias do Instituto Oswaldo Cruz & 2001 & 15 \\
\hline 1,20 & Memórias do Instituto Oswaldo Cruz & 2003 & 14 \\
\hline 1,20 & Memórias do Instituto Oswaldo Cruz & 2000 & 12 \\
\hline 4 & Memórias do Instituto Oswaldo Cruz & 2003 & 11 \\
\hline
\end{tabular}




\begin{tabular}{|l|l|l|l|}
\hline $1,20,22,24$ & Brazilian Journal of Medical \& Biological Research & 2001 & 9 \\
\hline 15,29 & Memórias do Instituto Oswaldo Cruz & 2004 & 9 \\
\hline 11 & Brazilian Journal of Medical \& Biological Research & 2004 & 8 \\
\hline $15,23,26$ & Anais da Academia Brasileira de Ciências & 2001 & 31 \\
\hline 4 & Memórias do Instituto Oswaldo Cruz & 2002 & 20 \\
\hline
\end{tabular}

Tabela 7 - Artigos Internacionais e Nacionais mais citados na Base de Dados SCOPUS

\begin{tabular}{|l|l|l|l|}
\hline Cód. Autores & Periódico & Ano & $\begin{array}{l}\text { No citaçóes } \\
\text { Scopus }\end{array}$ \\
\hline 23 & Nature & 2000 & 192 \\
\hline 6 & International Journal of Impotence Research & 2005 & 147 \\
\hline 6 & New England Journal of Medicine & 2007 & 140 \\
\hline 6 & Urology & 2003 & 124 \\
\hline 4 & AIDS Research and Human Retroviruses & 2001 & 64 \\
\hline 24 & Journal of Clinical Microbiology & 2001 & 53 \\
\hline 6 & Urology & 2001 & 53 \\
\hline 24 & Current opinion in infectious diseases & 2005 & 52 \\
\hline 7 & Journal of pediatric and perinatal nutrition & 2003 & 50 \\
\hline 24 & Infection and Immunity & 2005 & 48 \\
\hline 4 & Journal of Acquired Immune Deficiency Syndromes & 2000 & 44 \\
\hline $15,23,26$ & Anais da Academia Brasileira de Ciências & 2001 & 35 \\
\hline 19 & Cadernos de Saúde Pública & 2003 & 29 \\
\hline 1,20 & Memórias do Instituto Oswaldo Cruz & 2000 & 24 \\
\hline 4 & Memórias do Instituto Oswaldo Cruz & 2002 & 20 \\
\hline 1,20 & Memórias do Instituto Oswaldo Cruz & 2003 & 19 \\
\hline 6 & Sáo Paulo Medical Journal & 2002 & 19 \\
\hline $1,13,20,22,24$ & Memórias do Instituto Oswaldo Cruz & 2003 & 17 \\
\hline $18,20,27$ & Memórias do Instituto Oswaldo Cruz & 2001 & 15 \\
\hline $22,24,28$ & Revista da Sociedade Brás. de Medicina Tropical & 2003 & 12 \\
\hline 4 & Memórias do Instituto Oswaldo Cruz & 2003 & 11 \\
\hline 19 & Revista de Saúde Pública & 2003 & 11 \\
\hline & & & \\
\hline
\end{tabular}

\section{Conclusão}

Conclue-se que, o objetivo principal da pesquisa foi alcançado ao ser constatado através de vários indicadores de qualidade científica, o impacto da produção científica entre os próprios pesquisadores. A comunidade científica do CPqGM publica em periódicos internacionais e nacionais especializados que são indexados em bases de dados internacionais e nacionais como exemplo, as bases de dados do Institute of Scientific Information -ISI e do Scientific Electronic Library on Line - SciELO, que lhes garantem uma visibilidade e um evidente nível de endogenicidade. 


\section{Referências bibliográficas}

COURA, J. R. Impact factor scientific production and aquality of brazilian medical journal. Memórias do Instituto Oswaldo Cruz, Rio de Janeiro, v. 98, n.3, p.293-7, 2003.

GLOWER, S. W.; BOWEN S. L. Bibliometric analysis of research published in Tropical Medicine and International Health 1996-2003. Tropical Medicine and International Health, v. 9, n. 12, p. 1327-1330, 2004.

MENEGHINI, R. Avaliação da produção científica e o Projeto SciELO. Ciência da Informação, Brasília, v.27, n.2, p.219-220, 1998.

O Projeto SciELO (Scientific Electronic Library On Line) e a visibilidade da literatura científica "periférica". Química Nova, v.26, n.2, p.155, 2002.

OLIVEIRA, E.B. Produção científica nacional na área de geociências: análise de critérios de editoração, difusão e indexação me bases de dados. Ciência da Informaçáo, Brasília, v.34, n.2, p.34-42, maio/ago, 2005.

SANTANA, C.M. de O. Estudo dos canais de comunicaçáo utilizados pela comunidade científica do Centro de Pesquisas Gonçalo Moniz-CPqGM/FIOCRUZ, SalvadorBahia/Brasil. 1999. Dissertação (Mestrado)-Programa de Pós-Graduação em Ciência da Informação, Universidade de Brasília-UnB, Brasília, DF, 1999.

SANTANA, C.M. de O.; SAMPAIO, A.M.F.V.; BOA MORTE, A. de A.; MAIA, J.L. de S. A publicaçáo de "papers" nas revistas científicas que causam impacto em uma comunidade científica na área de Doenças Infecciosas e Parasitárias, no período de 2000 a 2004. Salvador, Bahia: ICML 2005. 